\title{
THE SYNDROME OF ACUTE CENTRAL CERVICAL SPINAL CORD INJURY
}

BY

\author{
RICHARD C. SCHNEIDER, JOHN M. THOMPSON, and JOSE BEBIN
}

From the Departments of Surgery and Sections of Neurosurgery of the University of Michigan Hospital, U.S. Veterans Hospital, and St. Joseph's Mercy Hospital, Ann Arbor, Michigan, and the Wayne County General Hospital, Eloise, Michigan, and the Department of Neuropathology, University Hospital, Ann Arbor

Any discussion of the diagnosis and treatment of spinal cord injuries inevitably involves controversial matters. Among the foremost of these are the indications for (Schneider, 1951, 1955) and the contraindications for (Schneider, Cherry, and Pantek, 1954) surgical intervention. It seems highly important, therefore, to establish criteria which will assist in determining whether an operation should or should not be undertaken. In 1955, a paper was published on "The Syndrome of Acute Central Cervical Spinal Cord Injury" (Schneider et al., 1954), a syndrome the presence of which contraindicated any surgical procedure. At that time, nine of the authors' cases which exhibited this neurological pattern were described in detail, and six others were abstracted from the literature. There was no pathological material presented, however, which would substantiate firmly the clinical thesis which had been advanced.

This paper is written to present 12 additional patients who exhibited the syndrome. In one case the post-mortem examination confirms the assumptions previously made on the basis of clinical examination. A study of this group of cases permits further consideration of the mechanisms involved in this type of injury.

The description of the syndrome reads:

"In acute cervical spinal cord injuries, there is a syndrome that suggests acute central cervical spinal cord involvement. It is characterized by disproportionately more motor impairment of the upper than of the lower extremities, by bladder dysfunction, usually by urinary retention, and by varying degrees of sensory loss below the level of the lesion. If the findings are caused by central cord destruction with bleeding, hematomyelia, there may be caudad or cephalad extension of the lesion with further progression of symptoms, perhaps culminating in complete tetraplegia or death. But if the symptoms are caused by concussion or contusion, with an edematous type of central cord involvement, there may be gradual return of function in a definite sequence. The amount of recovery depends on the degree of edema present compared to the extent of true destruction of nervous tissue. The lower extremities tend to recover motor power first, bladder function returns next, and finally strength in the upper extremities reappears, with the finer finger movements coming back last. The varying degrees of sensory impairment do not follow any set pattern of recovery."

It was first thought that the acute central cervical spinal cord injury was found only in severe hyperextension injuries. The importance of suspecting such an injury of the cervical spine in the presence of facial lacerations or contusions of the forehead $\overline{2}$ has been emphasized by Taylor and Blackwood (1948). When the hypertrophic arthritic cervical spine is hyperextended, there may be simultaneously compression of the anterior cord caused by a posteriorly placed bony spur and a posterior impingement upon the cord caused by a wrinkling of the ligamentum flavum. This mechanism was effectively demonstrated in myelography on cadavers by Taylor (1951). In younger specimens there was no distortion of the pantopaque column in the cervical spinal canal when the head was placed in a flexed or neutral position. When the cervical spine was forcibly hyperextended, however, there was definite encroachment posteriorly upon the pantopaque column in the spinal canal. Taylor suggested that this was probably caused by a bulging forward of the ligamentum flavum. When a cervical myelogram was made similarly in hyperextension in an elderly patient with posterior hypertrophic spurring of the cervical vertebral bodies, the bony spur anteriorly and the wrinkled ligamentum flavum posteriorly encroached simultaneously in the canal.

There are other mechanisms which may result in a squeezing of the cervical spinal cord. The acute central cervical spinal cord injury syndrome may occur in severe cervical compression fractures and in some cervical fracture-dislocations. It is difficult to postulate in cervical compression fractures whether the cervical spinal cord is squeezed in hyperextension or in flexion of the cervical spine. 
Cases of cervical fracture-dislocation, however, are a different matter. The acute central cervical cord injury syndrome may occur in an acute flexion injury accompanied by fracture-dislocation. The cervical spinal cord is acutely squeezed anteriorly by the lower involved, or caudad, vertebral body of a cervical fracture-dislocation and posteriorly by the lamina of the upper, or cephalad, involved vertebra.

The 12 patients presented here were admitted to four different hospitals in the year following the publication of the article on the acute central cervical cord injury syndrome (Schneider et al., 1954).

\section{Case Reports}

Case 1.-On May 3, 1955, S. B., a 63-year-old woman, fell headlong downstairs, striking upon her face. The patient developed complete tetraplegia accompanied by numbness of the lower extremities. Within two hours after the fall she was examined at Wayne County General Hospital, and the following positive findings were noted: a large contusion on the left forehead, slight paresis of the lower extremities, marked weakness of the upper extremities, and complete paralysis of the hands. There was a complete areflexia with no pathological reflexes. Hypaesthesia was noted from the shoulder downward. Position sense was intact. A radiograph of the cervical spine showed hypertrophic spurring at the $\mathrm{C}_{3}-\mathrm{C}_{4}, \mathrm{C}_{4}-\mathrm{C}_{5}$, and $\mathrm{C}_{5}-\mathrm{C}_{6}$ interspaces.

Several hours after she was admitted, her legs began to move readily, and on the following day they were strong. The upper extremities, however, showed almost complete paralysis of the forearm and hand muscle groups. Twenty-four hours after admission the hypaesthesia which had been present below $C_{7}$ bilaterally began to diminish gradually in the lower extremities. It was necessary to insert an indwelling catheter in the bladder.

A lumbar puncture on May 5, 1955, produced cerebrospinal fluid with 21 R.B.C.s per high-power field and a total protein value of $83 \mathrm{mg}$. per $100 \mathrm{ml}$. The blood Kahn test was 3 plus, but the spinal fluid Kahn test was negative. A jugular vein compression test showed no block. Twelve days after the injury movements in the patient's hands began to improve. By May 18, bladder control was good. A myelogram performed three weeks after injury with the patient in a steep Trendelenburg position showed that the pantopaque did not pass the level of $C_{6}$ until the neck was flexed. A defect was present at the $\mathrm{C}_{3}-\mathrm{C}_{4}$ interspace on the lateral view with a "step ladder" pattern on the anterior-posterior view.

By July 7, 1955, the patient moved her hands only weakly, but all other muscles of the extremities were normal. Pyramidal tract signs were observed in the left lower and upper extremities.

Case 2.-J. D., 55 years old, fell headlong down 23 steps on January 22, 1955. He remembered striking upon his forehead and left temple so that his neck was thrown into marked hyperextension. He was completely numb and paralyzed from the neck downward at the time when he was admitted to a local osteopathic hospital. Twenty- four hours after he was injured he could move the thumbs and toes. It was necessary to insert an indwelling catheter into the bladder. The strength in the lower extremities increased much more rapidly than in the upper, so that within a period of two weeks he could manipulate his lower extremities quite well. He could raise his arms to a certain extent. All forearm movements were poor, however; and, although his thumbs could be wiggled slightly, he could not move his fingers.

The patient was treated with intermittent halter traction until the time of his transfer to the U.S. Veterans Hospital, Ann Arbor, Michigan, on February 10, 1955. When he was admitted, a disproportionately better movement was noted in the lower extremities than in the upper, and his hands were very weak. Hypalgesia and hypaesthesia were observed over the upper extremities and, in a lesser degree, over the trunk and lower extremities. The deep reflexes were symmetrically hyperactive and no pathological reflexes were apparent. Vinke tongs were inserted. Radiographs of the cervical spine demonstrated a hypertrophic arthritis with spurring at all the interspaces but no evidence of fracture-dislocation.

A follow-up examination was made on March 4, 1956. His gait was impaired because of weakness in dorsiflexion of the right great toe and foot. Strength was less in the upper extremities than in the lower ones. The small hand muscles on the right side were atrophied. Vibration and position sensations were intact. The remainder of sensation had returned in patches. There was a zone of hyperaesthesia from $T_{10}$ through $T_{12}$ dermatome bilaterally. Hypalgesia was demonstrated over the dorsum of the right foot, leg, and knee, and bilaterally below the $\mathrm{T}_{12}$ dermatome. The deep tendon reflexes were more hyperactive in the right upper and lower extremities than in those on the left. There were definite pathological reflexes in both upper extremities and in the right lower, with equivocal abnormality in the left lower extremity.

Case 3.-L.R., 61-year-old farmer, was working on his manure spreader on Friday, April 13, 1956, when he fell headlong to the ground forcing his neck into extreme hyperextension. He could not move his arms or legs. After a short time he found he could move his legs weakly and attempted to roll over, but he was unable to do so. Four hours later the patient was taken to the hospital. He felt sharp shooting pains in his arms and legs. Upon sensory examination, however, there was a complete loss of sensation in both upper extremities. He could flex and extend his lower extremities and could move his shoulders but he could not move his forearms, wrists, or fingers. He had no pathological reflexes. Position sense was absent in both upper and lower extremities. Vibration sense was present in the left upper extremity, but absent over the remainder of the body. Complete thermanaesthesia was demonstrated below the level of $T_{1}$ dermatome bilaterally. He had hypalgesia over the left $\mathrm{C}_{6}$ and $C_{7}$ dermatone and hypalgesia to $L_{3}$ on the left and $\mathrm{T}_{12}$ on the right side.

Radiographs of the cervical spine showed extensive degenerative and hypertrophic changes. No evidence of any fracture-dislocation was observed. Dr. Adam 
McClay, the orthopaedic consultant, made a diagnosis of acute central cervical cord injury and stated that the prognosis was good. He advocated conservative treatment.

An examination on April 15 showed that the sensory pattern was essentially unchanged. The patient had powerful quadriceps and flexor movement in the lower extremities but still no detectable movement in the upper ones.

Case 4.-On March 23, 1956, H. S., a 51-year-old man, fell head first down a long flight of stairs while under the influence of alcohol. He regained his senses six hours later in a Detroit hospital, suffering from numbness and tingling from the neck downward over his body, marked weakness of his arms, paralysis of his hands, and slight paresis in his right leg. He had right-sided hyperactive deep tendon reflexes and a right extensor plantar reflex. A lumbar puncture was made within the first 24 hours after his injury, and the cerebrospinal fluid contained 60 red blood cells and 3 white blood cells per high-power field. The protein content of the fluid was $117 \mathrm{mg}$. per $100 \mathrm{ml}$. He had no bladder control during the first 48 hours in hospital. The motor power in his legs improved markedly within the first 24 hours, but he still had marked paresis in the right arm and moderate weakness of the left hand. A diagnosis was made of " conversion hysteria, possibly superimposed on some cord damage".

On the sixth day after injury, the patient was transferred to Wayne County General Hospital. Radiographs of the cervical spine demonstrated posterior hypertrophic spurs on $C_{6}, C_{5}$, and $C_{6}$ vertebral bodies, with no apparent evidence of fracture-dislocation.

On April 6, 1956, two weeks after he sustained the injury, the patient had only $5 \%$ normal strength in the muscle groups of the right arm. He had only minimal motion of the right thumb and fingers. The muscle groups of the left arm showed about $75 \%$ normal strength, in contrast to the lower extremities, where there was minimal paresis bilaterally. With the exception of the hypalgesia below the left knee, a sensory examination demonstrated normal responses. He had no biceps reflexes, but triceps reflexes were present, the left being more active than the right. The lower extremities had hyperactive deep tendon reflexes, the right quadriceps reflex being more active than the left. There were bilateral extensor plantar reflexes. After four days, he showed marked improvement in the strength of the right arm, although the hand grip was still weak.

Case 5.-E. B., a 52-year-old lumberman, fell 12 feet from a loaded pulp truck on the morning of March 2, 1956. He was unconscious for about an hour and did not recall in what position he had struck the ground. Upon regaining consciousness he found that he was temporarily unable to move all four extremities; his toe movements returned almost immediately, however. Within four hours he recovered motor power, first in the left leg, then in the right, so that he was able to stand erect four hours after injury. He could move his arms but they were much weaker than his legs. Finally, he was able, with assistance, to walk 250 feet to a car in which he was taken to a local hospital. Radiographs of the spine were taken and he was put to bed. No lumbar puncture was made.

During the first seven weeks in hospital the patient felt numb from the neck downward over his body, and was unable to use his arms, although he moved his legs slightly. Three and a half months after his injury he noted a generalized tingling sensation below the level of $T_{10}$ dermatome anteriorly and $T_{8}$ dermatome posteriorly. He began to walk unassisted about four months after the accident. His chief complaint was that he felt hot, prickly, and swollen sensations in the right leg. An indwelling catheter had to be used during the first two months of his stay in hospital until the bladder regained its function.

The patient was transferred to the University of Michigan Hospital, where the following neurological findings were noted: weakness in the legs and trunk, more marked paresis in the upper extremities, and maximal impairment of strength in the hands. There was hypaesthesia along the ulnar aspect of the right hand and a hypalgesic level at $T_{3}$ segment bilaterally. Dysaesthesia was found over the right leg. He had no vibratory sensation over the ankles, but position sense was present in both feet. With the exception of the Achilles reflexes, all deep reflexes were symmetrically hyperactive. Bilatera pyramidal tract signs were demonstrated in both uppero extremities and he had an equivocal extensor plantarn reflex in the right lower extremity. Radiographs of the cervical spine showed hypertrophic vertebral body spurring at $\mathrm{C}_{3}-\mathrm{C}_{4}$ and $\mathrm{C}_{5}-\mathrm{C}_{6}$ interspaces.

Case 6.-At 11.30 p.m. on October 11, 1955, J. H., ae 72-year-old man, fell from a wagon, striking on his head He was not unconscious but he was immediately paralyzed? in all four extremities with numbness from the neck downward. While in transit to the University Hospital in the ambulance, he began to move his toes and legs. When he was admitted to the hospital, the patient was alert and cooperative. He had no movement in the upper extremities or in the intercostal musles, but there was some motion in the hamstrings, quadriceps, adductors of the hips, and dorsiflexor muscles of the feet. On the left side from the $T_{2}$ to $L_{3}$ dermatome, he had a zone of loss of sensation to pinprick and dysaesthesia of temperature, and a loss of all sensory modalities of the remainder of the trunk and extremities. The deep reflexes in the extremities were equal, although symmetrically hyperactive in the legs. He had no pathological reflexes. A diagnosis was made of acute central cervical cord injury at $\mathrm{C}_{3}-\mathrm{C}_{4}$ or $\mathrm{C}_{4}-\mathrm{C}_{5}$ interspaces. Halter traction was applied.

Radiographs of the cervical spine demonstrated a fracture of the $C_{2}$ and $C_{3}$ spinous processes, and showed also some mild evidence of hypertrophic spurring of the vertebral bodies at $C_{4}-C_{5}$ and $C_{5}-C_{6}$ interspaces, with slightly more lipping at the $\mathrm{C}_{6}-\mathrm{C}_{7}$ interspace (Fig. 1). A lumbar puncture was performed and when jugular compression was applied, the cerebrospinal fluid rose and fell normally. Vinke tongs were substituted for the halter traction and, within 10 hours after admission, intercostal function had returned. A cystometric 


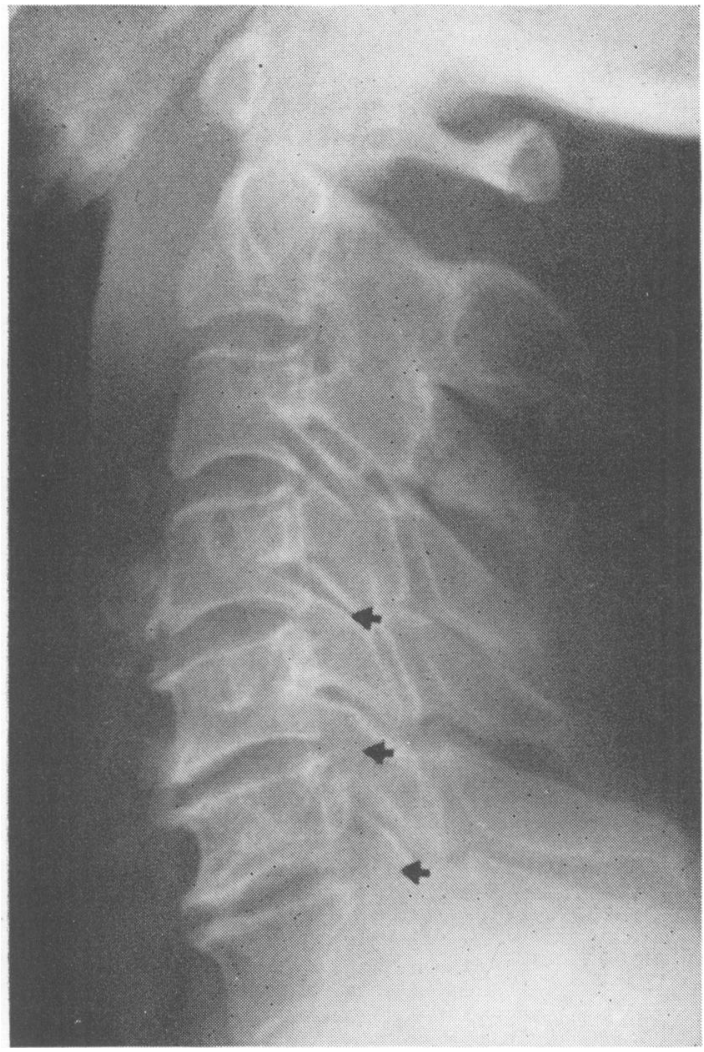

Fig. 1.-Case 6: Lateral radiographs of the cervical spine demonstrating fractures of $\mathrm{C}_{2}$ and $\mathrm{C}_{3}$ spinous processes and mild spurring at the $\mathrm{C}_{4}-\mathrm{C}_{5}$ and $\mathrm{C}_{5}-\mathrm{C}_{6}$ interspaces with more marked lipping at the $C_{6}-C_{7}$ interspace.

examination demonstrated a $180 \mathrm{ml}$. capacity reflex type of bladder on October 15, and the examining urologist concluded that the patient might be experiencing some return of function. Five days later there were uninhibited contractions suggesting a neurogenic reflex type of bladder. On October 25 bladder sensation partially returned. The bulbo-cavernous reflex was intact. The strength in the patient's legs improved but his arms remained paralyzed. On October 30 , his temperature rose to $103^{\circ} \mathrm{F}$. and his blood pressure fell. The chest radiograph showed right atelectasis and pneumonia. The electrocardiograph was normal. The patient became progressively worse and died on November 9. He had sustained a pulmonary embolus complicated by atelectasis and pneumonia.

Necropsy Examination.-This disclosed more marked bony ridges at the $\mathrm{C}_{4}-\mathrm{C}_{5}$ and $\mathrm{C}_{6}-\mathrm{C}_{7}$ interspaces than were demonstrable radiologically. The surface of the spinal cord appeared to be normal, but the cord was soft (Fig. 2). Cross sections of the cervical spinal cord taken at the upper $\mathrm{C}_{4}$ segment revealed, macroscopically, a fairly normal cord, somewhat flattened transversely and soft to palpation (Fig. 3). Cross section of the spinal cord just below this level exhibited necrosis of the central portion which extended toward the posterior surface to include parts of the posterior and postero-lateral funiculi. Sections above and below this segment exhibited only pallor of the funiculi.

Sections of the spinal cord were cut and prepared histologically with haematoxylin and eosin, Masson trichrome, and Weil stains. The sections of the lower fourth cervical segment of the spinal cord showed a large destructive lesion, which, at its maximum, included the central portion of the grey commissure, the anterior horns, the posterior horns, and the posterior columns of the cord (Fig. 4). A few millimetres below this level, the lesion appeared well circumscribed in the central portion of the cord immediately behind the central canal (Fig. 5). The lesion included the more proximal portions of both posterior horns and the medial parts of the posterior columns and the postero-lateral columns.

Microscopic examination of these areas showed almost complete destruction of the substance of the cord with liquefaction and necrosis. This area was filled with

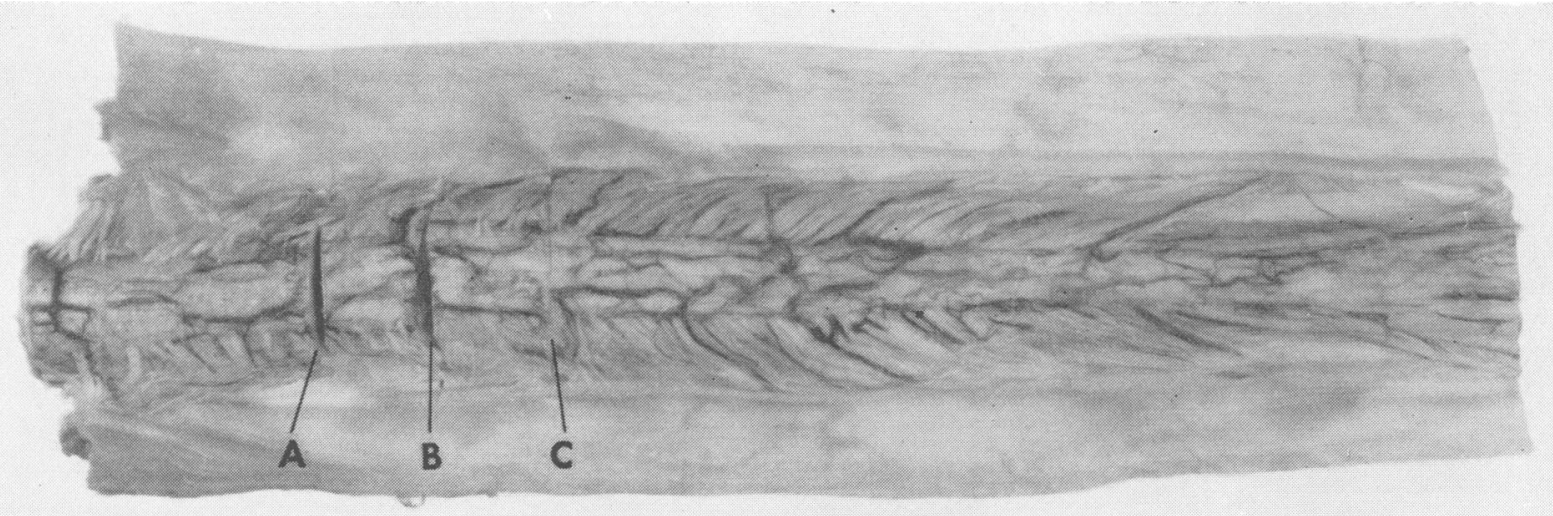

FIG. 2.-Case 6: The necropsy specimen showing that there was actually little evidence of gross external damage apparent to the dorsal surface of the cervical spinal cord. The three incisions $A, B$, and $C$ through the involved area unfortunately were made before photographing the specimens. 
FIG. 3.-Case 6: This shows the microscopic section of the cervical spinal cord at the upper $\mathrm{C}_{4}$ level $(A)$. The general pattern shows little evidence of destruction to the cord itself.
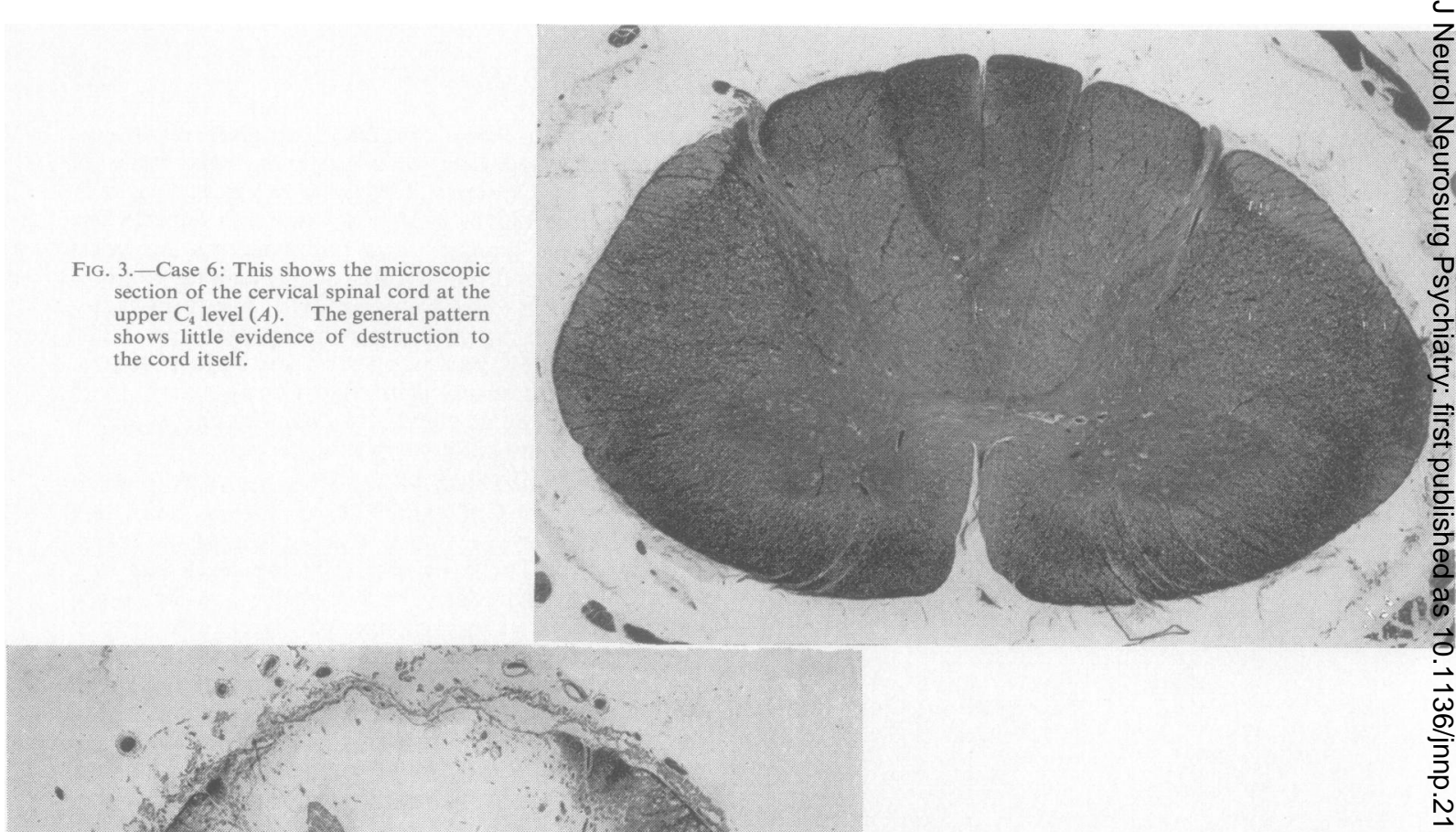

FIG. 4.-Case 6: A section taken at the lower C $7 \dot{\omega}$ segment $(B)$ demonstrating an intact pia mater $\bar{O}$ N but complete destruction of the cord centrally $\frac{\vec{D}}{\vec{\sigma}}$ and dorsally in the posterior columns. The 0 destruction spread into the lateral columns $\overrightarrow{\mathbb{D}}$ 음 leaving isolated islands of intact fibres. The $\varrho$ preservation of the more peripheral or lower $\bar{Z}$ extremity portions of the lateral cortico-spinal tract indicates the basis for the symptoms and $\delta$ 음 neurological findings in this syndrome. There is
no thrombosis of the anterior spinal artery.

Fig. 5.-Case 6: The microscopic section of the upper portion of the $\mathrm{C}_{5}$ cord segment $(C)$ shows central necrosis and destruction extending above the central canal and laterally toward the lateral corticospinal tract on the right side There is a patent anterior spinal artery. 
debris and numerous macrophages (gitter cells). The limits of the lesion were not well delineated from surrounding areas. Numerous small arterioles and newlyformed blood vessels were seen in this area. Many of these had hyalinized walls and appeared markedly congested. The blood vessels were more abundant in the transition zone between the necrotic centre and the surrounding cord tissue. The anterior horn cells were diminished in number and those remaining showed marked retrogressive changes. In the area of maximum involvement the anterior horns had completely disappeared and had been replaced by a mass of gitter cells, debris, and newly-formed blood vessels. At the marginal zone of the cord there was some degeneration of the myelin sheaths in the posterior and postero-lateral columns. The myelin degeneration of the posterolateral columns was more prominent in the deeper portions of the columns and merged into the gross lesion.

The leptomeninges were thickened with fibroblastic changes. The meningeal vessels, including the anterior spinal artery, posterior spinal arteries and veins, appeared to be normal but congested. The dura was normal in appearance.

Sections of the cord one segment below and one segment above the lesion showed marked congestion and moderate oedema. There was some loss of myelin in both postero-lateral columns, mostly in the deep portions. The anterior and posterior horns appeared well preserved in relation to the number of cells. The neurones showed changes compatible with ageing. The leptomeninges likewise exhibited changes of marked congestion and moderate oedema.

Case 7.-R. D., a 36-year-old severely paranoid schizophrenic male patient at the Veterans Hospital in Battle Creek, Michigan, was assaulted by another patient on January 21, 1954, and, during the ensuing scuffle, struck his head on the floor. The physician who examined him shortly afterwards stated that the patient was livid, had an irregular, thready pulse, and was incontinent of faeces. As the patient gradually regained consciousness, he complained of headache and pain radiating down the neck. There was no abnormality noted when the neurological examination was made. About 2 a.m. on January 22, 1954, the patient stated that he could not move, but he actually was seen moving his extremities. At 4 a.m., the patient could void only very small amounts, and later he was incontinent of a large amount of urine. On January 23, two days after the injury, he was unable to move either arm voluntarily. He also had a loss of pain and temperature sensation in the left arm, hyperactive patellar and ankle jerks, and a temperature of $104 \cdot 8^{\circ} \mathrm{F}$. By the next day he had marked weakness and flaccidity of the upper extremities but could move his legs. A lumbar puncture disclosed a clear cerebrospinal fluid with no block shown by the jugular compression test. Cervical spine films made on January 26 demonstrated a "crown" compression fraction of the $C_{5}$ vertebral body.

The patient was transferred to the Veterans Hospital in Ann Arbor, Michigan, and on admission was seen to have flaccid paralysis of both upper extremities with normal tendon reflexes, weakness in both legs, with spasticity and increased tendon reflexes and bilateral ankle clonus. On February 6, 1954, a neurosurgical consultant made a diagnosis of hyperextension injury of the cervical spine with compression fraction of $\mathrm{C}_{5}$ vetrebral body and central cord destruction or haematomyelia. By February 24, 1954, he walked with assistance. In March the patient had regained bladder control; and by May, 1954, he could feed himself.

Two years after the accident he exhibited more weakness in the upper than in the lower extremities, a spastic gait, a slight decrease in vibration sense in the right upper and lower extremities, symmetrically hyperactive reflexes, and a left extensor plantar reflex.

Case 8.-At 8.30 p.m. on July 9, 1954, E. S., a 12-yearold boy, dived into two feet of water and sustained an immediate paralysis of the upper extremities, but retained minimal movement in the legs. On admission to St. Joseph's Mercy Hospital, Ann Arbor, Michigan, he had only movements at the shoulders with no extension of the upper extremities or motion of the fingers. He had slight internal rotation in the thighs and the toes of both feet moved slightly. A level of hypaesthesia and hypalgesia was demonstrated extending to the $T_{7}$ dermatome bilaterally but motion and position sense were preserved. He had equally hyperactive deep reflexes. No extensor plantar reflex could be demonstrated. Priapism was present.

Radiographs of the cervical spine revealed a fracturedislocation of $\mathrm{C}_{2}$ vertebral body anteriorly on $\mathrm{C}_{3}$ and compression fractures of $\mathrm{C}_{4}, \mathrm{C}_{5}$, and $\mathrm{C}_{6}$ vertebral bodies with protrusion of the $C_{5}$ centrum posteriorly into the spinal canal. The posterior rim of the axis was in close proximity to the occipital bone.

A diagnosis of acute central cervical cord injury was made. A guarded prognosis was given to the patient's parents but the chance of recovery was considered good with the classical pattern of motor return predicted. The surgeon inserted Vinke tongs on the night of admission. By July 11, the priapism had subsided and there were bilateral extensor plantar reflexes. On July 12 a lumbar puncture revealed a bloody fluid (traumatic tap) without evidence of block when the jugular vein compression test was made. Five days later the patient had slightly better dorsiflexion in the left foot than in the right. On July 26, the day before transfer to another city, a cystometric examination revealed a reflex neurogenic bladder.

Reports from the Mary Free Bed Hospital in Grand Rapids, Michigan, where the patient was observed during his convalescence, indicated that he was able to move his fingers on August 17. By January 25, 1955, he could walk unassisted. At the time of his discharge on July 7,1955 , he had some slight residual weakness in his hands, but he performed all necessary functions readily.

Case 9.-C. L., a 52-year-old taxi driver, was examined at St. Joseph's Mercy Hospital, Ann Arbor, Michigan, on August 21, 1955, having been transported 500 miles without the benefit of any type of cervical immobilization. He had sustained an injury in an automobile accident five days before he was brought to the hospital. 
He had been rendered unconscious immediately, had a lucid interval, and then became semiconscious for 24 hours. His lower arms and hands were totally paralyzed, but he could move his lower extremities slightly. During the five days before his transfer, he improved markedly. Upon arrival in Ann Arbor, there was minimal strength in the grip and in the arms, but he had good motor power in both legs. There was hypalgesia over the $\mathrm{C}_{6}, \mathrm{C}_{7}$, and $\mathrm{C}_{8}$ dermatomes bilaterally and the entire right side of the trunk. The deep reflexes were symmetrically hypoactive with no pyramidal tract signs in the upper or lower extremities. Cystometric examination was normal. A diagnosis of acute central cervical spinal cord injury was made, and the prognosis was stated to be good.

Radiographs of the cervical spine showed a marked anterior fracture-dislocation of the $\mathrm{C}_{5}$ vertebral body anteriorly on the compressed vertebral body of $\mathbf{C}_{6}$. There were also fractures and posterior displacement of the laminae of $C_{5}$ and $C_{6}$ bilaterally, and a fracture of the $\mathrm{C}_{4}$ spinous process. Skeletal traction was applied. Decompressive laminectomy was deferred until August 31,1955 , to permit improvement in the patient's general condition. At operation the laminae of $C_{4}, C_{5}$, and $C_{6}$ vertebrae were found to be fractured and displaced posteriorly. The dura was opened, the arachnoid was sectioned laterally, and after the dentate ligaments were cut, the cord pulsated readily. The dura was closed tightly and spinal fusion was performed. He was discharged from the hospital on December 11, 1955. Radiographs of the cervical spine after fusion showed that the graft was calcifying nicely. Three months later the patient was able to return to his job as a taxi driver and was bothered only by some stiffness of the neck.

Case 10.-J. D., a 17-year-old student, was admitted to the University of Michigan Hospital on August 4, 1955 , one hour after diving into shallow water. He was paralyzed immediately in all four extremities and had to be carried from the water. On admission to the hospital the patient had diaphragmatic respirations. There was a complete areflexic tetraplegia with a sensory level at the $\mathrm{C}_{7}$ dermatome bilaterally and the patient was unable to void. Head halter traction was applied immediately, and radiographs demonstrated a subluxation of the $\mathbf{C}_{5}$ vertebral body anteriorly on the slightly compressed $\mathrm{C}_{6}$ vertebra. Thirty minutes after the application of the cervical traction he experienced a complete return of sensation. The halter traction was replaced by a Vinke skeletal tractor. A lumbar puncture revealed a complete block on the jugular vein compression test.

Twelve hours after he was admitted to the hospital, the patient had a decompressive laminectomy of $C_{5}$ and $C_{6}$ vertebrae. The right $C_{5}-C_{6}$ facet was locked. The dura was opened in the midline, the dentate ligaments cut, and the subluxation reduced by traction and careful prying with an osteotome. The pulsating cord appeared slightly hypaeremic and swollen. The dura was not closed.

Within 24 hours after the operation had been performed, the patient began to move his left foot and superficial as well as deep sensation returned on his right side. Four days after the injury he still had hypalgesia to the
$\mathrm{C}_{7}$ dermatome. Deep pain and position sensations were intact in the lower extremities. He showed marked recovery of motor power in the legs, but less in the upper and forearm muscles, and no return of function in the fingers or triceps muscles. The deep reflexes were equal and active and he had bilateral extensor plantar reflexes. The first finger movement appeared on August 16, and cystometric examination showed a normal bladder. The patient left the hospital on October 18 with his neck supported by a Forester collar. On December 9, 1955, he walked readily without a cane, and his hand function, which was the greatest residual deficit, was $65 \%$ of normal.

Case 11.-I. W., a 40-year-old man, dived into the Detroit River on August 8, 1955, and had to be pulled from the water because of total paralysis in all four extremities. He was admitted to the City of Detroit Receiving Hospital at 7 p.m. He had a complete tetraplegia with deep reflexes that were equal and active in the upper extremities, but the right lower extremity was areflexic. On catheterizing the bladder at 12 p.m., $800 \mathrm{ml}$. of urine was removed. Radiographs of the cervical spine revealed the $\mathrm{C}_{4}$ vertebral body dislocated anteriorly on $\mathbf{C}_{5}$. Skeletal tong traction was applied and a 35-lb. weight added. Serial radiographs of the cervicato spine were taken of the facets. There was complete reduction of the cervical fracture-dislocation and good realignment of the cervical spine so that the weight was़े reduced to $10 \mathrm{lb}$. The patient had no proprioception, $\mathfrak{a}=$ level of hypalgesia to the $\mathrm{C}_{3}$ dermatome bilaterally, an complete areflexia.

On August 10, a lumbar puncture showed no evidence of blockage of the subarachnoid space on the jugulas compression test. The spinal fluid was clear with onle $\vec{f}$ 4 red blood cells per high-power field and a total protein ${ }^{+} \infty$ level of $102 \mathrm{mg}$. per $100 \mathrm{ml}$. At midnight of the same day the patient had respiratory distress and difficulty in swallowing. It was decided that surgical intervention was not indicated.

On August 28, the patient had a slight return of motor power and sensation in the lower extremities. By September 4 , these functions were markedly improved. The skeletal tongs became disengaged on September 10, and it was thought advisable to discontinue their use. By September 19, 1955, the patient had regained some degree of motor power in both the upper and lower extremities, exclusive of hand movement. The indwelling catheter was removed.

At the time he was transferred to Wayne County General Hospital on September 22, 1955, he definitely had greater strength in the legs than in the upper arms, but the fingers and hands were still paralyzed. Pain and touch sensations were normal, but he had no position sense in the fingers of his right hand. The deep tendon reflexes were equal and active in the arms, but hyperactive in the legs. He showed bilateral Hoffmann responses and extensor plantar reflexes. The patient rejected the cervical spinal fusion operation which was recommended, and was discharged in a Taylor back brace with a Forester collar, on October 21, 1955.

On March 6, 1956, examination showed that the 
patient had weakness in all four extremities, but there had been progressive improvement in strength, and that now he could move his hands and fingers. Sensation was normal in all modalities. He had persistent pyramidal tract signs in all four limbs, with sustained left ankle clonus. Cervical spinal fusion was again advocated and this operation was performed on March 13, 1956.

Case 12.-Mr. X, a 68-year-old man, stumbled and struck his head and neck on the sidewalk on November 25,1955 . He was admitted to his community hospital where he complained of pain in the neck which radiated into both arms and hands. He also had paresis of the left arm and leg. Radiographs of the cervical spine demonstrated a fracture-dislocation of $\mathrm{C}_{5}$ vertebral body anteriorly on $\mathrm{C}_{6}$. Skeletal tong traction was applied and a 20-lb. weight added.

On the day after he was injured the patient required catheterization. Three days after injury he began developing mental disorientation, which was believed to be caused by cerebral anoxia secondary to decreased respiratory function. On December 2, 1955, he complained of severe pains in his arms and legs. A nurse's note stated on December 6 that the patient had fairly good control of his legs and often moved them but that he had less power in his arms. The right fingers and toes "were stiff". He continued to cry out because of severe pain in the extremities, and both physicians and nurses interpreted this as a result of mental confusion. On December 8,1955 , the patient had severe chest pain with associated dyspnoea. By that time all motor power had been lost in the lower extremities, but pain was still present. An electrocardiogram two days later showed a right bundle branch block. He continued to have severe discomfort throughout the entire trunk and extremities.

On New Year's Day, 1956, the skeletal tong traction was removed, and six days later, the patient had pain in the feet, legs, and arms and " he talked only of pain". Flexor spasms occurred in both knees but he had some voluntary movement in both, and marked improvement in feeling in both lower extremities. By January 10, 1956, the patient could move both lower extremities, had good motor power in the shoulders, poor elbow extension, fair motion in the forearm, but had no finger movement whatever on the left side.

The patient was transferred to Wayne County General Hospital in January, 1956. The indwelling catheter was removed. There were muscle contractures in both elbows, hips, knees, and to a lesser extent, in both hands, making evaluation difficult. Radiographs of the cervical spine, four months after trauma, demonstrated complete reduction of the dislocation with calcification to the vertebral body both anteriorly and posteriorly.

An examination on April 6, 1956, demonstrated that the patient's strongest movements were in the lower extremities in plantar and dorsiflexion of the feet. The other muscle groups of the lower extremities were weak. He had slight weakness in both forearms with marked paresis in the hand grip bilaterally. Hypalgesia was noted below the $T_{8}$ dermatome on the right. Touch was intact. Position sense was absent in the toes. Vibration sense was present in the knees. He had normal biceps reflexes, no triceps reflexes, and symmetrically hyperactive patellar and Achilles reflexes. Positive extensor plantar reflexes were demonstrated.

\section{Discussion}

Mechanisms.-In recent years an increasing amount of interest has been focused on the problems of chronic anterior cervical cord compression by hypertrophic spurs (Bedford, Bosanquet, and Russell, 1952; Brain, Northfield, and Wilkinson, 1952; Kahn, 1947; Spillane and Lloyd, 1952; Symonds, 1953), but too little attention has been devoted to acute traumatic lesions of the cervical spinal cord in such cases.

Case reports 1 to 6 concern patients who had had hypertrophic cervical arthritis and developed acute central cervical cord injury on the basis of hyperextension injury to the cervical spine. In Cases 1, 2,3 , and 6 , the patients had suffered definite facial or forehead injuries, supporting the belief that hyperextension injury had occurred. The patient in Case 4 had been drinking; and in Case 5 the man had been rendered unconscious, and therefore an accurate history concerning this specific point could not be obtained.

All these case reports demonstrate how acute central cervical spinal cord injury may occur in hyperextension of the hypertrophic arthritic spine without compression fracture or fracture-dislocation, giving support to Taylor's (1951) thesis. They should also serve as a warning against a too vigorous hyperextension of the cervical spine in the intubation of elderly patients for the administration of intratracheal anaesthesia. Reports have appeared in the literature calling attention to contingent dangers, and one of the authors (R. C. S.) is presenting elsewhere a case in which such an anaesthetic technique resulted in acute central cervical cord injury, though the patient gradually recovered.

The patient in Case 7 sustained a "crown". shaped cervical compression fracture, and it could not be determined whether or not this injury occurred with hyperextension or flexion of the cervical spine.

The syndrome probably resulted from acute flexion injury to the cervical spine and spinal cord in Cases 8 to 12. The patient reported in Case 8 had multiplemild compression fractures and fracturedislocations resulting in a "step" type of injury with multiple points of simultaneous anterior and posterior compression of the cervical spinal cord. In Case 9, an acute flexion injury must have occurred, accompanied by a severe fracture-dislocation of the cervical spine, along with a momentary holding of the laminae of the more cephalad vertebra. This 
squeezed the cord. An associated avulsion of the laminae must also have been present, causing compression of the cord, with the result that it was spared from complete transection. The fracturedislocations in Cases 10 and 11 are the disruptions which are commonly found and result in a simple squeeze of the cord rather than in transection.

In Case 12 the patient suffered initially a fracturedislocation of a hypertrophic cervical spine with little immediate neurological deficit. After the fracture-dislocation was reduced, however, the cord was probably drawn into some hyperextension over the hypertrophic spur so that an acute central cervical cord injury syndrome gradually developed. After skeletal traction was removed, the patient began to regain function following the characteristic recovery pattern.

Age.-A glance at the ages of these patients is instructive. In Cases 1 to 6 , the ages were 63, 55, $61,51,52$, and 72 years, respectively. As might be expected, these were the individuals with hypertrophic cervical spines who sustained acute central cervical cord injuries in hyperextension, without fracture or fracture-dislocation. The ages in Cases 7 to 11 were $36,12,52,17$, and 40 years, respectively, a much younger group as a whole. These individuals exhibited the syndrome in association with compression fracture or fracture-dislocation, more than possibly resulting from an acute flexion mechanism. The patient reported in Case 12 was 68 years old but his case was exceptional in that there was a fracture-dislocation of a hypertrophic cervical spine.

Anatomy.-In the cervical spinal cord there is a definite segmentation or pattern of fibres within the lateral corticospinal tract. The lumbar fibres, or those which supply the lower extremities, lie laterally in the cervical spinal cord. The thoracic or trunk fibres are in a medial position, while the cervical or upper extremity fibres assume a still more medial location with the finger fibres placed centrally. When the cervical spinal cord is squeezed from an anterior and a posterior position simultaneously, there may be a central zone of cord destruction surrounded by a more peripheral one of spinal cord oedema. The degree of permanent disability which these patients incur will depend upon the extent of central cervical cord damage, so that as the more peripheral zone of oedema recedes, the more peripherally located fibres in the lateral corticospinal tracts and adjacent tracts will regain function. As a result, the legs will regain function first, then bladder function returns, the arms recover next, and finger movements are regained last.

In order to study the various stresses which occur when the spinal cord is subjected to anteriorposterior compression, a sponge rubber model was constructed by Pantek (Schneider et al., 1954). Upon this model had been painted a diagram of the cervical spinal cord and its various fibre tracts. About its periphery was a fine rim of firmer rubber which served to represent the pia mater and the points of attachment of the dentate ligaments. The model was then placed into a wooden vice so that an anterior and posterior compressing force could be applied. By placing a grid over the model, it was possible to measure types and degrees of distortion. The study revealed the presence of at least four definite stresses, the predominant one being-as one might expect-the anterior-posterior compressive stress (Schneider et al., 1954).*

The use of this model obviously has its limitations, for no observations can be made concerning the influence of stress on the vascular components of the cord, the comparative looseness of texture near the vicinity of the central canal versus the compactness of fibre bundles near the periphery, or the fact that many of the sensory fibre tracts lie near the periphery of the cord so that they are affected merely by oedema rather than undergoing complete destruction. Ideally, the use of strain gauges in experimental animals would be an excellent method of evaluating these stresses but at the present time even the smallest of these is too bulky to be used in experiments with animals. The employment of the sponge rubber model and an analysis of the clinicas study which has been published elsewhere have given us only a small part of the information which we require.

Pathology.-For many years physicians have sought an explanation as to how the cervical cord can be damaged with apparently no attendant injury to the cervical spine. Holmes (1915) discussed this problem, stating that if the spinal cord were concussed, there might be either no gross evidence of cord damage or a uniform swelling opposite the point of impact of the cord against the bone. $\mathrm{He}$ suggested that since the spinal cord lay in a waterjacket of cerebrospinal fluid, it might oscillate to and fro in the fluid when the trauma occurred. These movements might become asynchronous with the motion of the cervical bony spine, and the spinal cord would then impinge upon the bone, causing concussion or contusion, and thus produce physical disturbance " especially in the fluid axioplasm of its fibres". Holmes observed that the greatest pathological change was a pronounced oedema in the affected segments, the most marked involvement

*The reader is referred to the original article for a complete discus sion of this problem. 
being found directly at the site of the blow. In his microscopic preparations of damaged cords, some of the fibre tracts had been destroyed but in the remainder of the fibres the myelin sheaths and axis cylinders were swollen. In the grey matter there were focal areas of softening, usually associated with haemorrhages which had peripheral zones of neuronal degeneration. These pathological findings could account for the presence of neurological signs which partially or completely disappear as severe oedema subsides. This is what occurs in the syndrome of acute central cervical cord injury.

Nerve fibres which have been compressed or squeezed are more likely to recover function than are those which have been acutely and severely flexed so that the neurons are "fractured" and permanent damage is sustained. A vascular impairment of the spinal cord is probable in patients thus affected. In Case 6 not only the central elements of the spinal cord but also the posterior columns were destroyed. Woodward and Freeman (1956) have shown that when the peripheral nerve roots and their associated neuronal sheaths were ligated over a zone of six segments (in dogs), central cavitation might appear along with some loss of the posterior columns. They attributed this loss to venous congestion in these regions. The posterior surface of the cord is drained by paired posterior veins which are not nearly as large and efficient in function as the large anterior venous channels. Woodard and Freeman's work has not proved conclusively that the changes were caused only by venous congestion, for certainly some degree of the arterial supply as well as the venous drainage must have been impaired when six peripheral nerve roots and their sheaths were ligated.

In Case 7, the delayed onset of the syndrome of acute central cervical spinal cord injury could scarcely have resulted from cervical cord oedema so long after the injury, and one must therefore consider the possibility of a traumatic thrombosis in the anterior spinal artery simulating the "late apoplexy" seen in traumatic cerebral lesions. There may be another alternative. The patient in Case 7 was psychotic; it is conceivable, therefore, that he could have suffered one injury which was followed by another episode of unwitnessed trauma some hours later, which accounted for his neurological signs.

\section{Treatment}

Lumbar Puncture.-This procedure should probably be used in almost all cases of spinal cord injury, and yet it is surprising to note how infrequently it is performed. The usual reason given for omitting the test is the fear of injuring the patient in manipulating him into a proper position for inserting the needle.

In our series of 12 patients, six had lumbar punctures. In the three hyperextension injuries which showed hypertrophic arthritis and in which spinal taps were made, there was no evidence of any block when the jugular vein compression test was done. This fits well with the data collected from our original series of nine cases in which there were four similar hypertrophic arthritis cases none of which involved subarachnoid space block. In the publication (Schneider et al., 1954) in which we reported these cases, it was suggested that there were several points of compression or "squeeze" of the cervical cord, distributing the oedema of the cord over several segments rather than localizing it at any single given point. This meant that one of the important criteria for operation, namely, complete subarachnoid block, was lacking.

In the remaining six patients of the series presented above, who may have sustained a cord injury associated with compression fracture or fracturedislocation of the cervical spine, three lumbar punctures were made. In two of them there was no block when the jugular vein compression test was carried out. (The patient in Case 7 had sustained a " crown type " compression fracture, and the individual in Case 8 had multiple compression fractures and "step" fracture-dislocations with multiple points of cord "squeeze".) One patient in this group (Case 10) showed a straightforward fracture-dislocation of $\mathrm{C}_{5}$ vertebral body anteriorly on $\mathrm{C}_{6}$. $\mathrm{He}$ had a complete block on manometric testing. It is interesting to note the examiner's observation: " Thirty minutes after the application of the cervical traction, there was complete return of sensation." One wonders whether repeated hourly taps would not have demonstrated a release of pressure and oedema and a subsidence of the complete block. This may have occurred in Case 11, where the fracture-dislocation was reduced early. Since the operative indication of complete block was present in Case 10, no chances were taken and the patient was treated by decompressive laminectomy. He subsequently made a fairly good recovery, but the question must be raised as to whether this would have happened if operative intervention had not been employed.

Myelography.-This test was undertaken only in Case 1. It is mentioned again here only to emphasize the danger of the procedure. Since it is always necessary to hyperextend the cervical spine in order to prevent the flow of radio-opaque material into the skull, there is a danger of increasing spinal cord damage, for it is in the hyperextended position that the original injury occurs. 
Operation.-The usual criteria presented for early operation upon patients with acute spinal cord injuries are complete block (as shown by the jugular vein compression test), an increase in neurological signs, or debridement of a compound fracture. In the majority of cases, and particularly in those where hypertrophic cervical spurring without fracture or dislocation of the cervical spine was found, these criteria were not present. In these individuals, anterior and posterior compression of the spinal cord existed simultaneously at several sites, so that no single point of spinal cord oedema or haematomyelia occurred and no subarachnoid block was usually to be observed. Damage to the cervical cord was acute. There was no progression of neurological signs but rather a regression, so that another of the customary indications for operation was absent. In the first patient discussed in our original presentation of this syndrome (Schneider et al., 1954), surgical intervention actually created a further compromise of the lateral cortico-spinal tracts which had already been damaged. When pressure was applied gently in order to retract the spinal cord, to section the dentate ligaments, and to explore anterior to the cord, the lower extremity components (which lie laterally in the pyramidal tracts) were pressed upon, and the lower limbs, which the patient had moved pre-operatively, were paralyzed after operation. Conservative treatment is the most satisfactory in patients with acute central cervical cord injury. These people may be placed in some type of traction applied directly in the plane of the body until the spinal cord oedema has subsided. This usually requires about four weeks, at the end of which time a collar may be substituted for the traction apparatus for a period of another six to eight weeks.

A single point of " squeeze" of the cervical spinal cord is frequently found in patients with an acute central cervical cord syndrome after flexion injury with fracture-dislocation of the cervical spine. A complete block on the jugular vein compression test is more apt to be found in such patients than in those who incur the syndrome in hyperextension. Such a situation occurred in Case 10, where there was a complete block, and immediate operation was therefore indicated. No lumbar puncture was made in Case 9, because the patient's generally poor condition precluded operation even though a block had been found. With the degree of fracture-dislocation shown radiologically, a complete block might certainly have been present. Patients who demonstrate the acute central cervical cord injury syndrome with associated fracture-dislocation need immediate operation, therefore, only if a subarachnoid block is found when the jugular vein compression test has been made. Otherwise, cervical skeletal traction should be applied in an attempt to reduce the deformity, and this should be followed by a spinal fusion some weeks later, after spinal cord oedema has subsided.

Myelotomy has been advocated in acute traumatic haematomyelia by Elsberg (1941), Holmes (1915), Cohn (1931), and others. Allen (1911), in one paper, and Freeman and Wright (1953), in another, have presented experimental evidence of symptomatic improvement in animals with cord contusions when pial incision and dorsal myelotomy was performed.

The present authors are inclined to agree with Frazier (1918). He had observed that in traumatic haematomyelia there were numerous haemorrhagic zones in the spinal cord which were not necessarily in continuity. Thus, myelotomy would damage an oedematous, but otherwise normal, spinal cord. McVeigh's (1923) observations, made on the basis of his experimental work on traumatic haematomyelia, tend to support Frazier's thesis of multiple areas of haemorrhage and destruction in the spinat $\omega$ cord.

Prognosis.-In the patients who show the acute central cervical spinal cord injury syndrome, the prognosis is usually good. As the cord oedema subsides, recovery follows the pattern described above. Power in the lower extremities returns first bladder function in the upper extremities next and finger movements last. If central destruction of the spinal cord is marked, only partial recovery may result. Any residual neurological deficit is most often present in the upper extremities with most marked impairment in finger movement.

The mere quiver of movement of a single muscle or the presence of deep reflexes in the lower extremity in spite of an otherwise complete motor and sensory loss has enabled the authors to give a guarded but reassuring prognosis to the family. They may be told that there is a fair chance of recovery of motor power in the lower extremities and perhaps even complete recovery of the entire body as well.

\section{Summary}

Twelve cases of the acute central cervical cord injury syndrome have been presented in detail. One of these cases affords the first pathological confirmation supporting the assumptions previously made relative to the syndrome. The importance of accurate diagnosis is stressed, with emphasis placed on the fact that operation is contraindicated, that the prognosis may be good, and that should recovery occur it will follow a definite pattern.

Any paper of this type, which embraces compilation 
of data from many sources, cannot be written without the cooperation of many individuals and allied hospital services. In the present instance, these include orthopaedic and radiology services as well as the neurosurgical. The authors wish to express their appreciation to the several staffs of the hospitals which aided in this work. In particular they are grateful to Dr. Will Glas, Department of Surgery, Wayne County General Hospital, for the opportunity of presenting cases from that institution. The authors also wish to thank Drs. James Hall and Adam McClay, of Munson General Hospital, Traverse City, Michigan, who have graciously supplied them with case material.

\section{REFERENCES}

Allen, A. R. (1911). J. Amer. med. Ass., 57, 878.
Bedford, P. D., Bosanquet, F. D., and Russell, W. R. (1952). Lancet, Brain, W. R. Rorthfield, D., and Wilkinson, M. (1952). Brain, $75,187$.

Brooke, W. S. (1944). J. Amer. med. Ass., 125, 117.

Cohn, E. (1931). Arch. Psychiat. Nervenkr., 95, 439.

Elsberg, C. A. (1941). Surgical Disease of the Spinal Cord, Membranes, and Nerve Roots. Symptoms, Diagnosis and Treatment. Hoeber. New York.

Frazier, C. H. (1918). Surgery of the Spine and Spinal Cord. Appleton, New York and London.

Freeman, L. W., and Wright, T. W. (1953). Ann. Surg., 137, 433.

Holmes, G. (19i5). Brit. med. J., 2, 769.

Kahn, E. A. (1947). J. Neurosurg., 4, 191

McVeigh, J. F. (1923). Arch. Surg. (Chicago), 7, 573.

Schneider, R. C. (1951). J. Neurosurg., 8, 360.

(1955). Ibid., 12, 95 .

Z, Cherry, G., and Pantek, H. (1954). Ibid., 11, 546.

-, Cherry, G., and Pantek, H. (1954).

Spillane, J. D., and Lloyd, G. H. T. (1952). Brain, 75, 177.

Symonds, C. (1953). Lancet, 1, 451.

Taylor, A. R. (1951). J. Bone Jt Surg., 33-B, 543.

Wo, and Blackwood, W. (1948). Ibid 30-B, 245.

Woodard, J. S., and Freeman, L. W. (1956). J. Neurosurg., 13, 63.

\section{THE MAY (1958) ISSUE}

The May (1958) issue contains the following papers:-

The Neuropathy of Multiple Myeloma. Maurice Victor, Betty Q. Banker, and Raymond D. Adams.

The Relapsing Course of Certain Meningiomas in Relation to Pregnancy and Menstruation. Edwin R. Bickerstaff, J. M. Small, and I. A. Guest.

A Case of Intramedullary Neurinoma. B. Ramamurthi, V. C. Anguli, and C. G. S. Iyer.

Recurrent Multiple Cranial Nerve Palsies. Sir Charles Symonds.

Finger Tremor in Tabetic Patients and its Bearing on the Mechanism Producing the Rhythm of Physiological Tremor.

A. M. Halliday and J. W. T. Redfearn.

Sensory Nerve Action Potentials in Patients with Peripheral Nerve Lesions. R. W. Gilliatt and T. A. Sears.

The Psycho-galvanic Reflex: A Comparison of A.C. Skin Resistance and Skin Potential Changes. J. D. Montagu.

Clinical Aspects of Susceptibility to Menthylpentynol. Edward Marley and Allen A. Bartholomew.

An Investigation of the Rosenow Antibody Antigen Skin Reaction in Schizophrenia. W. P. Gurassa and H. H. Fleischhacker.

Multilocular Cystic Encephalopathy of Infants. L. Crome.

Book Reviews.

A number of copies are still available and may be obtained from the Publishing Manager, British Medical Association, Tavistock Square, W.C.1, price 17s. $6 d$. 